\title{
Re: Nanotechnology in Urology
}

\author{
Jin S, Labhasetwar V
}

Lerner Research Institute, Department of Biomedical Engineering, Cleveland Clinic, Cleveland, USA

Urol Clin North Am 2009;36:179-88. doi: 10.1016/j.ucl.2009.02.005.

\section{EDITORIAL COMMENT}

Nanomedicine is a new scientific revolution that interests in nanoscale materials for health science. At nanoscale, the physical properties of materials are changeable as well as their interactions with cells and tissue. Nanomedicine can be useful in the diagnosis and treatment of human diseases at cellular and molecular levels using engineered nanodevices and nanostructures. As an example of this imaging technique, the development of tumor-targeted contrast agents (such as superparamagnetic iron oxide and iron oxide nanoparticles) based on nanoscale materials are becoming more popular for the development of target-specific magnetic resonance imaging agents. Other applications on nanomedicine are detection of single nucleotide polymorphisms in genes related to cancer and genetic diseases. On the other hand, it can be promising for bacterial detection in many infection diseases. Moreover, application of nanotechnology for treatment are based on nanocarrier delivery of drugs for the treatment of urooncological diseases (such as nanocarrier-based delivery of anticancer drugs to tumor tissue) or nononcological diseases (such as treating erectile dysfunction with prostaglandin-E1). In the near future this technique can be used for delivery of genes (such as nanoparticle mediated gene transfection). The other logical idea about application of nanotechnology can be possible solution for tissue engineering in end-stage renal diseases or neobladder reconstruction. In the future, nanomedicine can provide many nanotechnological equipments for the diagnosis and treatment of urologic diseases.

Fehmi Narter, MD, PhD

๑Copyright 2018 by the Association of Urological Surgery / Journal of Urological Surgery published by Galenos Publishing House. 